Four years later he counted fingers at $2 \mathrm{ft}$. with one eye and at 6 in. with the other. No paralysis of any muscle.

4. The Gradual Improvement in Sight which may Occur. M. S., girl, aged 5 years, an attack of severe lead ocular neuritis in 1899. Five dioptres of swelling in the discs, and much neuro-retinitis encroaching on the macular regions. Paralysis of right external rectus; paresis of left external rectus. Recovered with damage to the retinae and some postneuritic atrophy of discs. A. year later counted fingers at 6 yards and saw ${ }_{80}$. Two years later right eye saw ${ }_{18}^{\circ}$, left eye a partly. Now (1905) sees right eye $\frac{8}{9}$, left eye $\frac{6}{12}$. Her age $3^{4}$ partly discounted to dises still pale and irregular. etwe. In each fundus the veins about the macular region are somewhat dilated. She concentric contraction, central vision being most acute.

A sister of this patient suffered from colic and very marked blue lines in her gums, also from twitching of shoulders and arms.

\section{Monocular Diplopia.}

E. M., a peculiarly intelligent gir] of 8 years, was admitted in E. M., a peculiarly intelligent gir of 8 years, was admitted in January, 1904, with a typical attack of lead neuritis, with lead in the urine and blue lines on the gums. She recovered quickly, had ultimately regained perfect vision. She had been ill for three weeks, and had squinted for one week before admission. On admission, she saw double with both eyes open and also with the right eye closed, but single with the left eye closed. The swelling of the discs only amounted to 2 dioptres. I noted that there appeared to be relatively more exudation in the horizontal meridian of the left fundus than in the vertical. examination left for two days later. The monocular diplopia had, however, then disappeared. It had lasted for seven days. I believe it to have been due to temporary astigmatism.

$$
\text { 6. A Chronic Case. }
$$

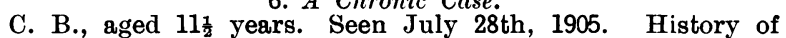
defective sight for last year or two; difficulty in seeing blackdefective sight for last year or two; difficulty in seeing blackboard at school ; reads partly; right eye a little the better; discs large and pale, with ill-defined edges and seen well with
$+2 \mathrm{D}$, whereas the general fundus did not bear a + glass; no $+2 \mathrm{D}$, whereas the general fundus did not bear a + glass; no
paralysis of any ocular muscle; nails bitten; skin of hands paralysis of any ocular muscle; nails bitten; skin of hands moist, even in winter; very distinct blue lines in gums round To stop biting nails, to stop school, and take iodide of potassium in 5-grain doses three times daily. October, 1905, tried $\frac{6}{6}$, and saw all of $\frac{6}{9}$. January, 1906, saw two letters of $\frac{6}{6}$; said to be in much better health; allowed to go to school. April, 1906, sees $\frac{6}{6}$. Discs do not bear a + glass; they are large and rather pale, with the edges slightly irregular, in a state, in fact, of slight post-neuritic atrophy; no difficulty in seeing at school. The slight optic neuritis in this case when first seen school. The slight optic neuritis in this case when first seen cases.

Treatment has consisted in removal from their homes, confinement to bed, dilute sulphuric acid and saline laxatives, and hypodermic injections of pilocarpin daily-the last continued for six weeks. After the first few days, iodide of potassium in the proportion of 5 grains thrice daily to a child of 8 years.

In cases with symptoms of encephalopathy I have found latterly that lumbar puncture and the draining of some 2 drachms of fluid is a help, and repeat it every few days if the first puncture gives indication of increased tension.

1 Australasian Medical Gazette, 1897. 2 Ibid., 1907. 3 Ibid., 1897. 4 BRITISH MEDiCal JodRnal, July 18th, 1908: Ophthalmoscope, August, 1908. SAustralasian Medical Gazette, 1904. 'Transactions, Australasian Medical Congress, Seventh Series, 1905

\section{A NOTE ON THE}

\section{INFLUENCE OF THE NERVOUS SYSTEM UPON INFECTIVE PROCESSES.}

By G. LENTHAL CHEATLE, C.B., F.R.C.S.,

SURGEON TO KING'S COLLEGE HOSPITAL AND ITALIAN HOSPITAL.

In previous publications which related to the influence exerted upon cancer by the nervous system I usually mentioned cases in which certain infective processes occupied the same nerve areas as the cancers under description.

In the present article I wish to enlarge on the mere statement of fact, and to add something further. 'The relationship between the nervous system and infective processes can be described under two heads-peurovascular and neurotrophic. I believe they are quite distinct from one another.

\section{Neurovascular.}

It must be admitted that in most cases the neurovascular system is normal immediately before the actual moment of inoculation, and that in most cases the changes which occur in the neurovascular system after inoculation are due to the presence of the infective processes. On the other hand, it is obvious that if abnormal vaso-constriction or abnormal vaso-dilatation were present at the time of the

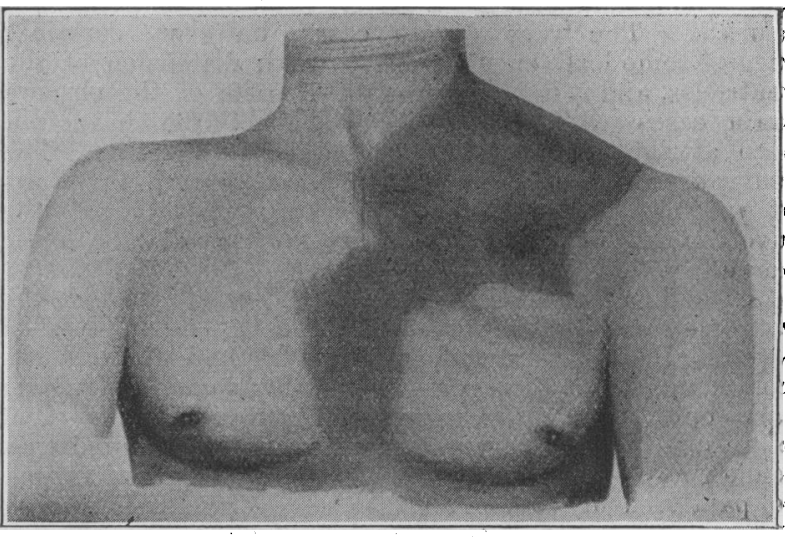

Fig. 1.-Area of ' neurovascular disturbance in a case of infection by Streptococcus pyogenes (Lenhartz).

inoculation, then the infective process might be modified by either of these conditions. However this may be, my object now is to point out that there is a marked change in the neurovascular system associated with some processes of infection.

The shaded area in Fig. 1 represents an intensely red skin due to the dilatation of blood vessels supplied by the posterior spinal root ganglion of the left third cervical nerve. The case is one of infection by Streptococcus pyogenes affecting the subcutaneous tissue of the chest,

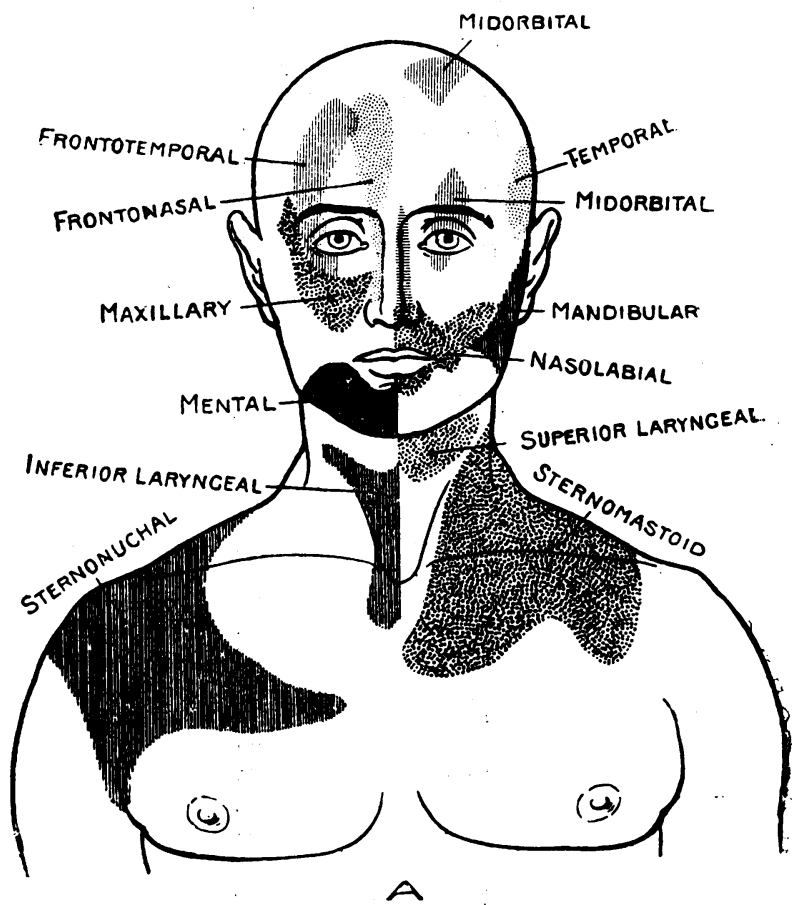

Fig. 2.-To show sterno-mastoid area. (With Dr. Henry Head's kind permission, this diagram is taken from his article in Brain. to the Pain of Visceral Disease.)

neck, and shoulder. Compare the red area in this case with the sternomastoid area in Fig. 2, which also corresponds to the area supplied by the posterior spinal root ganglion of the third cervical nerve. It is impossible to state the exact mechanism by which the blood vessels of this area. became dilated. It may have been due to the direct irritation caused by the presence of the micro-organism in the posterior root ganglion, or by a reflex act induced by stimulation of the peripheral nerves. It is very unusual 
to see vascular dilatation of a whole posterior spinal root area in infective cases. As a rule, the redness bears no relationship to any particular central distribution, and is purely peripheral in type. Can the presence of the vaso-

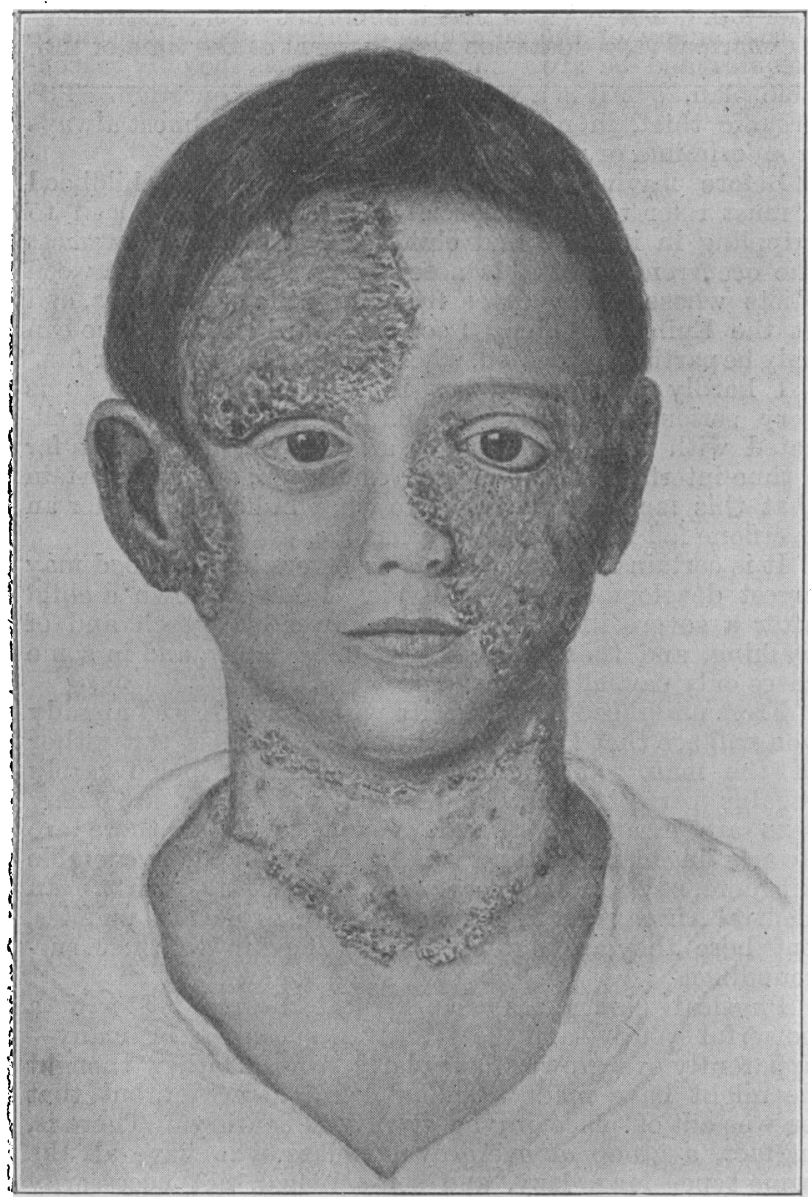

Fig. 3.-Distribution of tuberculosis in neck and head.

dilatation be the cause of the limitation to nerve areas which I have demonstrated in certain infective diseases? For example, see in Fig. 3 the limitation of the tuberculosis in the neck. I feel sure that changes of purely vascular dilatation have not much influence in the limitation of local infective processes to nerve areas. Were vascular dilatation capable of such powers of limitation, one would expect to see it most marked in those cases in which the infected processes were limited to definite nerve areas. But this is not so; the limitation to a nerve area is most common in the more chronic infective processes in which there is no marked vaso.dilatation in the area diseased nor in the parts surrounding that area. I do not wish to be understood to say that vaso-dilatation has no effect in limiting the spread of infective processes. I have no doubt that vaso-dilatation does act beneficially in these cases. All I venture to point out is that vaso-dilatation is not a marked feature of those infective diseases in which limitation to nerve area is most obvious, and that, therefore, there is some factor of limitation to take into consideration in these cases which is not purely vaso-dilatation.

\section{Neurotrophic.}

Fig. 3 shows the distribution of tuberculosis in neck and head of a child. The case is taken from the collection of the Polyclinic, with the kind permission of Captain Pinch, I.M.S. The distribution of the disease in the neck corresponds to the same area seen in the neck in Fig. 4, which shows the area of cutaneous tenderness which Dr. Head observed in the neck of a man who was suffering from an attack of angina pectoris.

There is no doubt that the area of occupation in the tuberculosis of the neck of Fig. 3 is the same as in Fig. 4.
Even the little notches in the centres of the lower margins are present in both figures. I regard the area occupied by the tuberculous neck in Fig. 3 as a neurotrophic area. There can be no doubt that it is surrounded by neurotrophic areas, and the neurotrophic situation can be summed up as follows: One of two things, or a combina. tion of both, must be operative-either there is a positive or negative influence of a neurotrophic nature in the normal areas which repels the onset of the disease, or there is a positive or negative influence of a neurotrophic nature the operation of which limits the disease to the whole of the neurotrophic area affected.

The situation is open to the following argument: The presence of the disease in the affected area is alone sufficient to account for its spread within and final limitation to that area, for the simple reason that presence of the disease lowers the immunity of the area in which it is operative. I see that this may be an important factor; but it is impossible to assume that, with the disease on their boundaries, the surrounding areas have not been attacked by it; and if they have been attacked, then they must have repelled it; and as they have repelled it, then the neurotrophic affairs cannot have been in identical states on the two sides of the boundaries even before the inoculation of the tuberculous area occurred. For if the neurotrophic conditions had all been the same before infection, there would not have been any limitation to a nerve area, but the spread of disease would have been equal in all directions except for mechanical obstruction. Hence the area diseased was more susceptible to the Bacillus tuberculosis than were the surrounding parts before inoculation occurred. There appears to me no doubt that there is a neurotrophic factor to be taken into consideration in the consideration of the process of immunity.

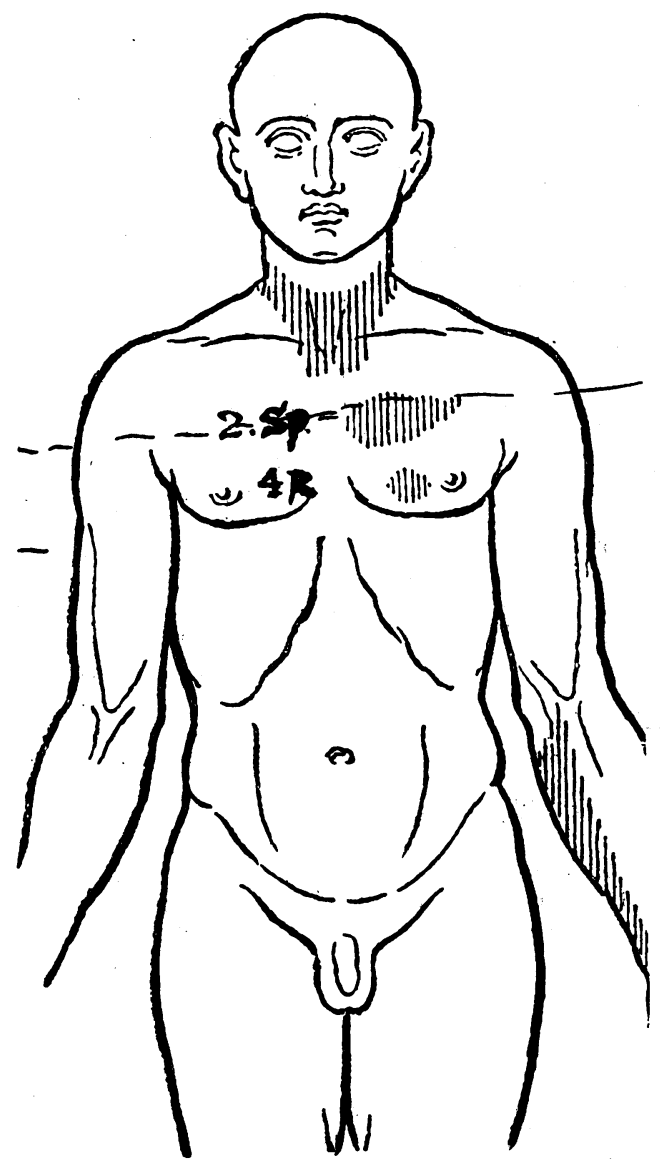

Fig. 4.- Area of cutaneous tenderness during anginal attack in a man with aortic disease. (Taken, with Dr. Head's kind

With regard to the other distributions of tuberculosis on the forehead and face in Fig. 3, they look very suspiciously like limitations to the superior and inferior divisions of the fifth nerves. 